\title{
Hospital information systems in the Slovak Republic
}

\author{
Anita Romanová ${ }^{1}$, Natália Švejdová ${ }^{2, *}$ \\ ${ }^{1}$ University of Economics in Bratislava, Faculty of Business Management, Department of Information \\ Management, 85235 Bratislava, Slovak Republic \\ ${ }^{2}$ University of Economics in Bratislava, Faculty of Business Management, Department of Information \\ Management, 85235 Bratislava, Slovak Republic
}

\begin{abstract}
Process-managed organization has much in common with the management of the information system. Management of the company can therefore be compared to the management of the system, while it is necessary to ensure information and communication flows. Effective information processing and knowledge base building are the basis for the functioning of the organization and its strategic intent. The means to achieve this goal is the information system. Informatization is the process of penetrating ICT into all areas of society in order to make it work effectively. An important part of building the information society is eHealth. The Internet, information systems, information technology and healthcare electronisation are important aids to improve and streamline the functioning of the entire health system, as they enable faster and more effective communication between all actors in the health sector (ie pharmacies, patients, healthcare facilities and insurance companies). The aim of this paper is to analyze hospital information systems in hospitals on the territory of the Slovak Republic, to identify the modules they consist of and to define interconnections of these systems with other information systems, not only within the given organization but also from the external environment.
\end{abstract}

\section{Introduction}

The planning, implementation and maintenance of information and communication technologies (ICT) in health care is a very demanding process. The health care sector shows certain specifics - lack of funds, substandard demands arising mainly from legislation and requirements of insurance companies and authorities (it is almost impossible to use standard information systems (IS) and therefore they are tailor-made, which is reflected in their financial demands), the need for safe and reliable ICT (not only from the IT point of view, but also patient-related safety - eg operating room devices and computers must meet stringent requirements and be treated with special preparations to avoid compromising patient health) or the need to process large volumes of data, which must be securely stored but accessible at any time to medical staff. Hospital information systems are complex

\footnotetext{
* Corresponding author: natalia.svejdova@euba.sk
} 
integrated systems that manage the operation of the hospital and manage all aspects of it, such as health, administrative, financial or legal. In practice, this involves replacing conventional paperwork with a computer system and thus simplifying the administrative work associated with healthcare facilities.

\section{Information systems and information technologies in healthcare}

Literary sources unambiguously document that the era of computer use in health services emerged in technologically and knowledgeable countries as soon as these devices appeared within the reach of medicine. So we can talk about half a century of history. The past 15 years are particularly significant. ICT development has accelerated, mainly due to the miniaturization of computer technologies and the dramatic decline in their cost. Instead of large mainframes, today we have them on our desktops in the form of personal computers, portable in briefcases (notebooks), and even in the pocket of a work jacket (PDA - a personal digital assistant in conjunction with a mobile phone capable of digitally capturing and transmitting image information).

Computers having high performance are utilized in many industries to increase competitiveness and quality. Informatization has become one of the critical factors determining the success of a company. Information technology has been heavily introduced in many companies, and the highly complex medical industry is no exception. [11] The development of computers and communication technologies in the 1990s can be seen as a breakthrough. In this period, funds were also created that enabled computers to be interconnected (even wirelessly) into information networks, and the potential of using the Internet in the health sector was not fully appreciated. [5] Therefore, it can be argued that it was in this period that a great information and knowledge potential arose - a system of information highways, which creates not only a significant knowledge, but also an organizational phenomenon. In the healthcare industry, information has a significant influence on service efficiency, physician decisionmaking and human health. The information of selfmedication consumer's behavior is not come from medical diagnosis and is not imposed by physicians. Good information integration are required for providing effective patient services. The healthcare industry should have the best information management because information has a grave impact on physician services. Information flows in the healthcare industry occur for the production of medicine and the manufacturing of medical supplies for hospitals, and they help organizations provide the right patient services. [9] The rapid development of ICT has improved not only the technical, but also the software, enabling the creation of a large database IS accessible to multiple users simultaneously in real time. However, the use of ICT in the health sector was not limited to communication and archiving records. New technologies enable medical devices to be integrated into computers, making them an integral part of healthcare delivery. These include computer and ECG, EEG, EMG, vital signs monitoring, laboratory testing equipment (hematology, microbiology, or biochemistry), or a computer-connected imaging device, such as a computer. X-ray, CT, sonograph and others.

Research of information systems and information technologies used in the field of health care is carried out by a large group of foreign experts. Ehrenfeld and Cannesson point out that the use of ICT in healthcare is essential. For health ICT they use the term health information technology (HIT), which is used to collect, transmit, display or store patient data electronically. HIT is also a concept that involves the use of computerized systems to make healthcare information available to patients, healthcare providers or insurance companies. Using HIT helps reduce doctors' mistakes, costs and paperwork, and increases the efficiency and quality of healthcare. [7] 
Sayles states that HIT includes a wide range of products, technologies and services such as remote and mobile medical technology, cloud services, medical devices, telemonitoring tools, assistive and sensory technologies, electronic health records (EHR) and others. These technologies allow users to collect, share and use medical information. [15] Although Health IT (HIT) has tremendous potential for improving quality and reducing cost in healthcare, significant challenges need to be overcome to fully realize this potential. [1]

HIT is the overarching term applied to various information and communication technologies used to collect, transmit, display, or store patient data. HIT, which includes hospital information systems (HIS), electronic health record (EHR), computerized physician order entry (CPOE), electronic prescribing (e-prescribing), clinical decision support systems (CDSS), and bar-coded medication administration (BCMA), has been widely implemented in healthcare organizations to support healthcare services. HIT is expected to increase the quality and safety of healthcare services by improving communication, patient delivery time, and documentation. Despite the benefits, HIT also introduce consequences when it is poorly designed, implemented, and used. Problems related to HIT can disrupt healthcare delivery, harm patients, and even cause death. [14]

In their research (2015), Cresswell and Sheikh confirmed that the use of HIT tools is widespread in healthcare facilities. Given the proven benefits of HIT, the use of these technologies is now essential. However, the balance between the benefits and risks of the use of information technology in healthcare facilities in the coming years is not clear. Therefore, in order to improve the planning and successful implementation of these technologies, they considered the need to use technology forecasting methods. [6]

HIT can effectively improve the quality and efficiency of medical services, distribution of health care resources, safety in health care, and output of scientific research. Therefore, governments of various countries have set up ambitious plans to develop HIT and invested enormous amounts of money in this development, using HIT as an important starting point for the reformation of medical services and medical systems. [10]

Almunawar and Anshari are also involved in health information system (HIS) research. In their research HIS: Concept and Technology (2012), they concluded that the use of ICT in healthcare facilities grew at the same pace as in other areas of industry or services. They state that there is a growing tendency for people to know and participate actively in the prevention and care of their health, and this fact leads to the development of information systems in health care. This trend leads to greater involvement of patients in taking information, decision-making and responsibility for their own health through ICT. This vision is achievable through shared care, which is based on health telematics networks and services, linking hospitals, laboratories, pharmacies, primary health care providers and patients through a "virtual health center", accessible via a single entry point online. [3] However, Hansen concluded that the major problems of HIS and HIT are the exchange of data between users as well as the actual use of data from health systems to provide intelligent decision support. [8]

The development of ICT, as well as artificial intelligence, will eventually manifest itself in medicine. Currently, there are already ITs that remember and effectively apply the knowledge of leading experts. They can therefore be called intelligent or knowledge-based systems, and their use can be found in the process of diagnosis, choice of medical procedures and decision-making processes. Such an intelligent system is e.g. Watson Health from IBM. It is a cognitive system with artificial intelligence, which is used to diagnose the patient's medical records and suggest the recommended treatment. Watson also suggests a treatment that is ineffective and also determines the probability of success / failure of the treatment. After assessing the patient's health status and suggesting a treatment procedure, the attending physician has available scientific articles from around 
the world for a more professional look at diagnostics from IBM Watson Health. IBM spent more than $\$ 6$ trillion on the development of this knowledge system.

One of the elements of the information system in healthcare facilities is HIS. Moghaddasi et al. in their study (2018) state, that HIS is an integrated information system that not only provides patient information but also supports all hospital activities, including clinical, administrative and financial activities. In addition, HIS plays an important part of the hospital budget. Usually 2 to $5 \%$ of the hospital operating budget is devoted to the information system. Studies also show that hospital staff spend most of their time recording information, but only $42 \%$ of their time is devoted to clinical activities. [13] Based on a foreign study carried out by a team of experts (Ahmadian, Dorosti, Khajouei, Gohari) in 2015, the human factor can be considered the largest and most important problem of hospital information systems. On the basis of this research, it emerged that HIS is failing because health professionals have a negative attitude towards IS and that there is no incentive to (effectively) use IS. [2]

HIS are often portrayed as precious tools which can enable better quality and safety of healthcare and make the whole process of patient treatment more efficient. HIS are expected to collect, store, analyse, and provide information about the patient to caregivers, thus, enabling better decision-maiking and timely actions with respect to treatments and the provision of healthcare services. Healthcare systems are best described as complex adaptive systems. [12]

The IS of healthcare facilities cannot be understood only on the health level, but these IS must also ensure the normal operation of the entire organizational unit. Last but not least, the IS must interconnect all internal departments and provide feedback, it must be able to adapt sufficiently to changing conditions in the internal and external environment of the company. However, efficiency in terms of cost and achieving the effects of its application is also important. The IS aims to improve the performance of people in organizations and businesses through the use of information technology. For this reason, it is necessary to regard IS in health care as a complex HIS (CHIS) ensuring the operation of the entire organization, including the provision of health care.

The use of ICT in the health sector can not only be viewed from an administrative perspective, but its full potential needs to be fully exploited. The achievements of the $21 \mathrm{st}$ century will not only improve health care and reduce healthcare costs, but also ensure more effective treatment or prevention, thus improving and extending people's lives. However, the fact remains that cognitive systems, which largely assist doctors in diagnosing and treating patients (such as Watson Health), are expensive, and few health care facilities (both in Slovakia and abroad) are able to do so. allow to procure.

\section{Research design}

The main aim of this paper is to analyze HIS, identify the modules it consists of and define the interconnection of HIS with other IS not only within the organization but also from the external environment.

We have analyzed this area by a questionnaire survey. The questionnaires were distributed to 40 respondents ( 38 state hospitals + two private health care providers).

When compiling the questionnaire, we started from the theoretical knowledge that we gained during the study of this selected issue, and we were also very helpful by experts in the field of hospital information systems, healthcare electronization, network management and personal data protection.

The return rate of the questionnaire was $75 \%$, which represented 30 respondents. We were unable to obtain data from private healthcare providers on the grounds that the questions in the questionnaire were very sensitive in terms of protecting business secrets, IT 
security or even corporate reputation. We were unable to obtain information from the group of state hospitals from 5 health care providers in order to maintain the information security of the organization or with reference to the optional provision of comprehensive information in the form of a questionnaire. 3 hospitals were eventually excluded from the statistical sample, as they currently only act as a social services home or diagnostic center.

The structure of the analyzed sample of 30 respondents is shown in the following table.

Table 1. Structure of respondents depending on the founder and territorial scope.

\begin{tabular}{|l|r|r|r|r|}
\hline \multirow{2}{*}{ Founder } & \multicolumn{2}{|c|}{ Region of the Slovak Republic } & \multirow{2}{*}{ Total sum } \\
\cline { 2 - 4 } & West & Middle & \multicolumn{1}{c|}{ East } & \\
\hline $\begin{array}{l}\text { Ministry of } \\
\begin{array}{l}\text { Health of the } \\
\text { Slovak } \\
\text { Republic }\end{array}\end{array}$ & $33.34 \%$ & $20.00 \%$ & $10.00 \%$ & $63.34 \%$ \\
\hline $\begin{array}{l}\text { Higher } \\
\text { territorial unit }\end{array}$ & $10.00 \%$ & $10.00 \%$ & $0.00 \%$ & $20.00 \%$ \\
\hline $\begin{array}{l}\text { Other } \\
\text { authority }\end{array}$ & $3.33 \%$ & $3.33 \%$ & $10.00 \%$ & $16.66 \%$ \\
\hline Total sum & $\mathbf{4 6 . 6 7 \%}$ & $\mathbf{3 3 . 3 3 \%}$ & $\mathbf{2 0 . 0 0 \%}$ & $\mathbf{1 0 0 . 0 0 \%}$ \\
\hline
\end{tabular}

When analyzing the data, it was necessary to differentiate hospital subjects into size categories. Whereas the European Commission Recommendation no. 2003/361 / EC in our case would evaluate 26 subjects as large enterprises and only 4 subjects as medium-sized enterprises, we decided to compile our own categorization of hospitals on the basis of basic characteristics of individual subjects.

The primary criterion for the classification of the subject is the number of employees. Based on our analysis, the closest approximation to this breakdown is the number of outpatient examinations $(\mathrm{r}=0.7562 ; \mathrm{r} 2=57.18 \% ; \mathrm{p}<0,001)$, then the number of hospitalizations $(\mathrm{r}=0.8031 ; \mathrm{r} 2=64.50 \% ; \mathrm{p}<0,001)$. Based on these relationships and data analysis using statistical indicators average and standard deviation, Slovak hospitals can be categorized based on the following indicators:

Table 2. Proposed criteria for categorization of Slovak hospitals.

\begin{tabular}{|l|c|c|c|}
\hline \multirow{2}{*}{ Indicator } & \multicolumn{3}{|c|}{ Category of organization } \\
\cline { 2 - 4 } & Small & Medium & Large \\
\hline $\begin{array}{l}\text { 1. Number of } \\
\text { employees }\end{array}$ & $\mathrm{X} \leq 178$ & $178<\mathrm{X} \leq 1446$ & $1446<\mathrm{X}$ \\
\hline $\begin{array}{l}\text { 2. Number of } \\
\text { outpatient } \\
\text { examinations }\end{array}$ & $\mathrm{X} \leq 15355$ & $15355<\mathrm{X} \leq 395196$ & $395196<\mathrm{X}$ \\
\hline $\begin{array}{l}\text { 3. Number of } \\
\text { hospitalizations }\end{array}$ & $\mathrm{X} \leq 2095$ & $2095<\mathrm{X} \leq 27797$ & $27797<\mathrm{X}$ \\
\hline
\end{tabular}

One of the above categories falls within a hospital subject if it has the relevant number of employees (the main criterion) and meets at least one secondary criterion (the number of outpatient examinations or the number of hospitalizations). Based on our proposed categorization, the respondents of the research sample can be classified as follows: 
Table 3. Categorization of respondents to the research sample.

\begin{tabular}{|l|r|r|r|}
\hline \multirow{2}{*}{ Founder } & \multicolumn{2}{|c|}{ Category of organization } \\
\cline { 2 - 4 } & \multicolumn{1}{|c|}{ Small } & \multicolumn{1}{c|}{ Medium } & $13.33 \%$ \\
\hline $\begin{array}{l}\text { Ministry of Health } \\
\text { of the Slovak } \\
\text { Republic }\end{array}$ & $13.33 \%$ & $36.68 \%$ & $0.00 \%$ \\
\hline $\begin{array}{l}\text { Higher territorial } \\
\text { unit }\end{array}$ & $0.00 \%$ & $20.00 \%$ & $0.00 \%$ \\
\hline Other authority & $3.33 \%$ & $13.33 \%$ & $\mathbf{1 3 . 3 3 \%}$ \\
\hline Total sum & $\mathbf{1 6 . 6 6 \%}$ & $\mathbf{7 0 . 0 1 \%} \%$ & \\
\hline
\end{tabular}

In the next sections of this paper we will start with this categorization of hospitals into small, medium and large organizations.

\section{Research results}

The first part of our questionnaire was focused on the identification of a hospital subject in order to categorize the organization. This was followed by a section dealing with basic information on HIS.

$83.33 \%$ of respondents stated that they have a HIS that was tailored to the requirements of the organization. The remaining $16.67 \%$ use standard HIS, ie. that they were not additionally customized by suppliers. In Slovak hospitals they use HIS, which were implemented on average in 2009 ( $M=2009$; $S D=6.61$; Min=1995; Max=2019). In practice, unfortunately, we may also encounter systems that are more than 20 years old (two respondents in our research), and these systems are very outdated today and often record their failure rate. 8 respondents of our research have a system not older than 3 years.

Only 3 Slovak hospitals, which are under the founding competence of the SelfGoverning Region of Trenčín have a unified HIS. This system was procured for all three hospitals at the same time, consisting of the same modules, extensions and functionalities. These entities perceive the advantages of a unified IS for the possibility of making uniform outputs from the IS for the purpose of management from the Self-Governing Region of Trenčín, the possibility of joint negotiations on contracts with suppliers, quantity discounts, the same system adjustments for all organizations.

Table 4. Number of workstations and users in Slovak hospitals.

\begin{tabular}{|c|c|c|c|c|}
\hline \multirow{2}{*}{ Organization } & \multicolumn{2}{|c|}{ Number of workstations } & \multicolumn{2}{|c|}{ Number of users } \\
\hline & $\mathbf{M}$ & SD & M & SD \\
\hline Small & 86 & 12 & 116 & 17 \\
\hline Medium & 337 & 218 & 589 & 371 \\
\hline Large & 792 & 237 & 1482 & 420 \\
\hline
\end{tabular}

From Table 4 we can see that in Slovak hospitals there are two users per workstation. In the case of medium and large organizations we see that a large number of people work with IS, and therefore these systems must constantly and quickly respond to user requirements, process and store a large amount of information (not only about employees, but also patients, assets, economic variables, etc.). 
In the next part of the questionnaire we focused on modules, which consist of HIS, IS that make up CHIS, to which specialized devices the HIS is connected and whether the IS of organizations are also connected with external IS.

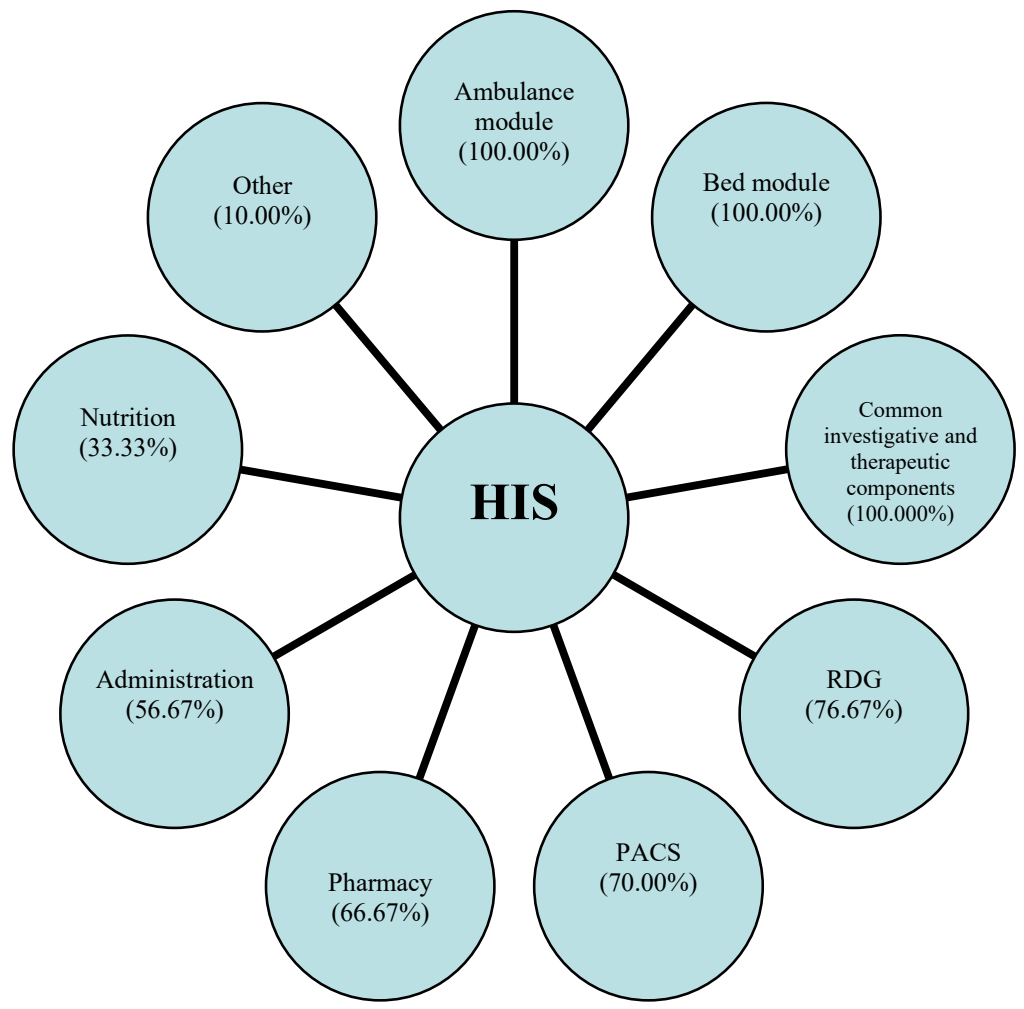

Fig. 1. Modules of hospital information system in Slovak hospitals.

Figure 1 shows which modules consist of HIS in Slovak hospitals. The ambulance, bed and Common investigative and therapeutic components modules are available to HIS at all health care providers. More than $50 \%$ of respondents identified administration, pharmacy, PACS and RDG modules.

PACS (picture archiving and communication systems) are clinical information systems used for the acquisition, archival, and post-processing distribution of digital images. An image must either be directly acquired using digital radiography or be digitised from a paper-based format. It can be stored using an electronic, magnetic, or optical storage device.[4]

Nutrition is part of HIS in one third of hospitals and $10 \%$ of respondents have other HIS modules (eg transfusion module, transport health service, boiler room and incineration system). 


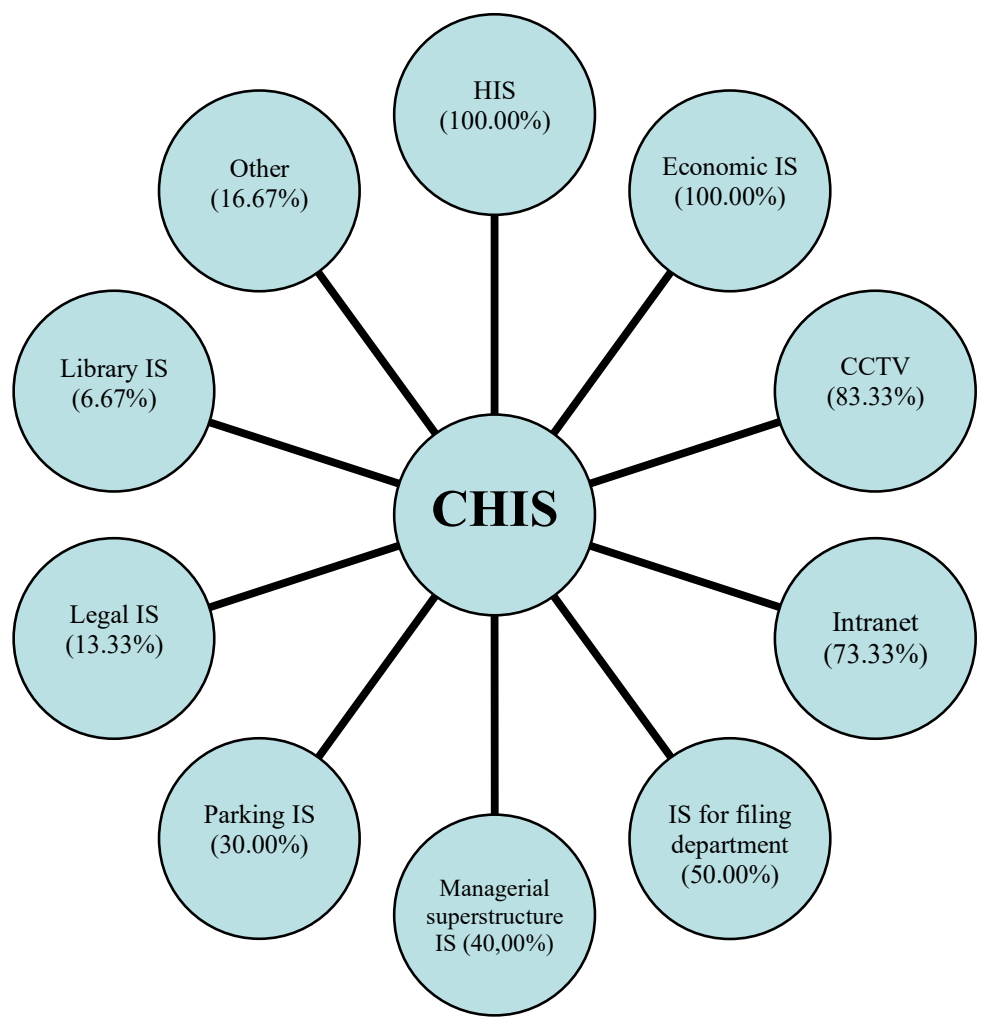

Fig. 2. Modules of complex hospital information system in Slovak hospitals.

As shown in Figure 2 for each respondent of our research, the CHIS module includes the HIS module and the economic IS. More than $70 \%$ of respondents have CCTV and intranet. $40 \%$ of hospitals have managerial superstructure and one third of organizations have a parking system. The least represented IS are legal IS (13.33\%) and library IS (6.67\%). As other modules of the CHIS respondents stated eg. attendance system for employees, electronic requests for purchase and repairs, waste records, questionnaire IS or IS for building management.

In the next part of the research we focused on whether HIS are linked to specialized facilities and therefore it is necessary that the IS not only communicates with these facilities, but also processes and evaluates the results of patients. The interconnection of HIS with such specialized devices was observed in $80 \%$ of respondents, the most common devices being: digital X-ray (77\%), analyzers (70\%), sono (53\%), CT (47\%), MRI (20\%) and central drug preparation plant (7\%). Two other respondents mentioned other specialized devices to which HIS is linked: sampling weights of hematology-transfusion department, cryo-bifuge, shock freezer, angiographic devices and special urological devices. None of our respondents has cognitive systems (eg IBM Watson).

The last area we analyzed is the interconnection of IS hospitals with IS from external environment. In all of the subjects monitored by us, we noted the link between CHIS and the IS ezdravie (IS of electronic health care in the Slovak Republic), the IS of individual health insurance companies and the IS of the National Center of Health Information. $60 \%$ of hospitals have links to the DôveraLab IS (the insurance company Dôvera, which provides access to the results of laboratory examinations) and $47 \%$ of the organizations are linked to the Dôvera Návrhy (hospital suggestions for spa treatment of patients). Two 
respondents reported linking CHIS via online applications to Medirex and Dr.Magnet and linking to external laboratories.

\section{Discussion and conclusion}

The informatization of health care is currently a basic trend that affects not only health care as a whole, but also individual facilities providing health care. Computerization not only facilitates the work and processing of documents by healthcare professionals, but also plays a major role in communication between doctors, patients, laboratories, hospitals and insurance companies. ICT that is applied in the health sector has the potential to contribute to improving the quality, cost-effectiveness, timeliness and mobility of health services. [12]

By carrying out our research, we managed to define the modules that make up the hospital information systems of individual organizations, as well as the interconnection of these information systems to other systems in hospitals (eg economic IS, parking IS, CCTV, etc.). The fact that CHIS are not closed IS within one organization was confirmed by an analysis of the connection of CHIS to external IS (IS of insurance companies, IS of electronic health care of the Slovak Republic, etc.). We have also been able to demonstrate that CHIS must also ensure the operation and processing of the results of specialized devices, such as: digital X-ray, sono or MRI. Based on these findings, we can confirm the claim of Moghadassi et al., that CHIS is an integrated information system providing not only patient information but providing support for all hospital activities (clinical, administrative, personnel, financial, etc.). Last but not least, these information systems must constantly respond to the changing environment (legislation, conditions of insurance companies and authorities).

A less satisfactory area is the area of interconnection of health care organizations and workplaces. It is e-health (in the territory of the Slovak Republic edzravie) that this problem should be eliminated. The implementation of this project requires that ICTs be installed in appropriate healthcare structures in appropriate structured network connections. The interconnection of IS between hospitals was recorded only in the case of three respondents using a single IS (these are hospitals within the founding competence of the Self-Governing Region of Trenčín).

The use and benefits of ICT in the health sector are certain. As domestic and foreign authors define the positives of the use of ICT resources in medicine, the risks associated with them should not be forgotten. In the health sector, sensitive personal data are processed, which need to be handled not only in terms of acquisition, but especially storage. ICT is a constantly changing field and it is therefore very important that healthcare providers plan their procurement, implementation and maintenance very well. As the ICT field is very dynamic - what is innovative today is already 'out of date' tomorrow - the spending of these modern means must be as efficient and effective as possible.

\section{Notation}

The paper was elaborated within VEGA No. 1/0388/20 IT Managemenet in Enterprises in Slovakia: International Standards and Norms Versus Individual Business Processed proportion $100 \%$. 


\section{References}

1. R. Agarwal, G. Gao, C. DesRoches, A. Jha, The Digital Transformation of Healthcare: Current Status and the Road Ahead. Information systems research. 21, 796-809 (2020)

2. L. Ahmadian, N. Dorosti, R. Khajouei, S. Gohari, Challenges of using Hospital Information Systems by nurses: comparing academic and non-academic hospitals. Electronic Physician. 6, 4625-4630 (2017)

3. M. Almunawar, M. Ansahri, Health Information Systems (HIS). Researchgate. (2012)

4. M. Alohali, F. Carton, Y. O'Connor, Investigating the antecedents of perceived threats and user resistance to health information technology : a case study of a public hospital. Journal of Decision Systems. 29, 27-52 (2020)

5. A. Black, J. Car, C. Pagliari, C. Anandan, K. Cresswell, T. Bokun, B. McKinstry, R. Procter, A. Majeed, A. Sheikh, The Impact of eHealth on the Quality and Safety of Health Care: A Systematic Overview. Plos Medicine. 8, e1000387 (2011)

6. K. Cresswell, A.Sheikh, Health Information Technology in Hospitals: Current Issues and Future Trends. Future Healthcare Journal. 1, 50-56 (2015)

7. J. Ehrenfeld, M. Cannesson, Monitoring Technologies in Acute Care Environments : A Comprehensive Guide to patient Monitoring Technology. (2013)

8. D. Hansen, Challenges and Opportunities for Health Information Systems Research. Health Information Science. 60-67 (2012)

9. S. Lancharoen, P. Suksawang, T. Naenna, Readiness assessment of information integration in a hospital using an analytic network process method for decision-making in a healthcare network. International Journal of Engineering Business Management. 12, $1847979019899318(2020)$

10. J. Liang, Y. Li, Z. Zhang, D. Shen, J. Xu, G. Yu, S. Dai, F. Ge, J. Lei, Evaluating the Applications of Health Information Technologies in China During the Past 11 Years: Consecutive Survey Data Analysis. JMIR Medical informatics. 8, e17006 (2020)

11. M. Liao, I. Lin, Performance Evaluation of an Information Technology Intervention Regarding Charging for Inpatient Medical Materials at a Regional Teaching Hospital in Taiwan: Empirical Study. JMIR mHealth and uHealth. 8, e16381 (2020)

12. V. Ljubicic, P. Ketikidis, L. Lazuras, Drivers of intentions to use healthcare information systems among health and care professionals. Health Informatics Journal. 26, 56-71 (2020)

13. H. Moghaddasi, et al. Hospital Information Systems : The status and approaches in selected countries of the Middle East. Electronic Physician. 5, 6829-6835 (2018)

14. L. Salahuddin, Z. Ismail, U. Hashim, N. Ismail, R. Ikram, F. Rahim, N. Hassan, Healthcare practitioner behaviours that influence unsafe use of hospital information systems. Health Informatics Journal. 26, 420-434 (2020)

15. N. Sayles, Health Information Management Technology: An Applied Approach. (2012) 